\title{
High frequency limit of the Transport Cross Section and boundedness of the Total Cross Section in scattering by an obstacle with impedance boundary conditions
}

\author{
Aleksenko A.I., J.P.Cruz, Lakshtanov E.L., ${ }^{* \dagger}$
}

\begin{abstract}
The scalar scattering of the plane wave by a strictly convex obstacle with impedance boundary conditions is considered. The uniform boundedness of the Total Cross Section for all values of frequencies is proved. The high frequency limit of the Transport Cross Section is founded and presented as a classical functional of the variational theory.
\end{abstract}

\section{Introduction}

Consider a strictly convex body $\Omega \subset \mathbb{R}^{3}$ with Lipschitz boundary $\partial \Omega$ and $k>0$. The scattered field is given by the Helmholtz equation and a radiation condition

$$
\begin{gathered}
\Delta u(r)+k^{2} u(r)=0, \quad r \in \Omega^{\prime}=\mathbb{R}^{3} \backslash \Omega \\
\int_{|r|=R}\left|\frac{\partial u(r)}{\partial n}-i k u(r)\right|^{2} d S=o(1), \quad R \rightarrow \infty
\end{gathered}
$$

with Dirichlet, Neumann or impedance boundary conditions of the form

$$
\left.\mathcal{B}_{\gamma}(u)\right|_{\partial \Omega} \equiv-\left.\mathcal{B}_{\gamma}\left(e^{i k\left(r \cdot \theta_{0}\right)}\right)\right|_{\partial \Omega}, \quad r=(x, y, z) \in \partial \Omega,
$$

${ }^{*}$ Department of Mathematics, Aveiro University, Aveiro 3810, Portugal. This work was supported by Centre for Research on Optimization and Control (CEOC) from the "Fundação para a Ciência e a Tecnologia" (FCT), cofinanced by the European Community Fund FEDER/POCTI, and by the FCT research project PTDC/MAT/72840/2006.

$\dagger^{\dagger}$ e-mail: lakshtanov@rambler.ru 
where $\gamma \geq 0$ is a constant, $\mathcal{B}_{\gamma}=(\partial / \partial n)-i k \gamma$ and $e^{i k\left(r \cdot \theta_{0}\right)}$ is an incident field formed by a plane wave with incident angle $\theta_{0}=(0,0,1) \in S^{2}$. In [4], for example, is proved the existence and uniqueness of the solution of (11)-(3). A function $u(r)$ which satisfies the mentioned conditions has asymptotic

$$
u(r)=\frac{e^{i k|r|}}{|r|} f_{\gamma}(\theta)+o\left(\frac{1}{|r|}\right), \quad r \rightarrow \infty, \quad \theta=r /|r| \in S^{2}
$$

where function $f_{\gamma}(\theta)=f_{\gamma}(\theta, k)$ is called scattering amplitude and measure

$$
\sigma_{\gamma}=\int_{S^{2}}\left|f_{\gamma}(\theta)\right|^{2} d \sigma(\theta)
$$

is called Total Cross Section. $\sigma$ is a square element of the unit sphere. The projection on the incident direction $\theta_{0}$ of the total momentum transmitted to the obstacle is given by a measure called Transport Cross Section (for a large volume normalization)

$$
R_{\gamma}=\int_{S^{2}}<\theta_{0}-\theta, \theta_{0}>\left|f_{\gamma}(\theta)\right|^{2} d \sigma(\theta)
$$

In case of Dirichlet (or Neumann1) boundary conditions the limit behavior of the scattering amplitude in the high frequency regime is described completely by following two statements: [1, Theorem 1]

$$
f_{0}(\theta)=\frac{1}{2} \mathcal{K}\left(y^{+}(\theta)\right)^{-1 / 2} e^{i k<y^{+}(\theta) \cdot\left(\theta-\theta_{0}\right)>}+O(1 / k), \quad \theta \neq \theta_{0} .
$$

Here $y^{+}(\theta) \in \partial \Omega$ is the preimage of $\boldsymbol{n}(\theta):=\left(\theta-\theta_{0}\right) /\left(\left|\theta-\theta_{0}\right|\right) \in S^{2}$ under the Gauss map, $\mathcal{K}\left(y^{+}\right)$is the Gauss curvature at $y^{+} \in \partial \Omega$. The estimation $O(1 / k)$ is uniform on compact subsets of $\left\{\theta \in S^{2} \mid \theta \neq \theta_{0}\right\}$.

The behavior near the forward directions is given by (see [6, 7$]$ )

$$
\lim _{k \rightarrow \infty}\left|f_{0}\right|^{2}=|f|_{c l}^{2}+\sigma_{c l} \delta\left(\theta_{0}\right)
$$

where $\left|f_{c l}(\theta)\right|^{2}=\left(2 K\left(y^{+}(\theta)\right)\right)^{-1}$ is the classical density of scattered rays, and the limit is in sense of distributions. This formula allows one to obtain limits at high $k$ of all measures like

$$
\int_{S^{2}} \varphi(\theta)\left|f_{0}(\theta)\right|^{2} d \sigma(\theta), \quad \varphi \in C\left(S^{2}\right)
$$

\footnotetext{
${ }^{1}$ To simplify expressions we will use only notation of $f_{0}(\theta)$ in formulas (6)-(9), but all of them are valid also for the case $\gamma=\infty$. See [2] for proof of $\lim _{k \rightarrow \infty} \sigma_{\infty}=2 \sigma_{c l}$
} 
In particularly we have,

$$
\lim _{k \rightarrow 0} \sigma_{0}=2 \sigma_{c l}, \quad \lim _{k \rightarrow 0} R_{0}=R_{c l}
$$

Here $\sigma_{c l}, R_{c l}$ are classical Total Cross Section and classical Transport Cross Section. Calculation of the limit of the $\sigma_{0}$ in the sphere case is mentioned in every student book of physics, and for the case of convex bodies there is a rigorous proof in [2]. Moreover, this fact was used in [6] to prove (77).

The case of impedance boundary condition (i.e. finite values of $\gamma>0$ ) is not completely studied. Exists an analog of (6) ([1, Th.1.]) namely,

$$
f_{\gamma}(\theta)=\frac{1}{2} \mathcal{K}\left(y^{+}\right)^{-1 / 2} e^{i k<y^{+}(\theta) \cdot\left(\theta-\theta_{0}\right)>}\left(\frac{\gamma-<\boldsymbol{n}(\theta), \theta>}{\gamma+<\boldsymbol{n}(\theta), \theta>}\right)+O(1 / k)
$$

uniformly on every open subset of $\left\{\theta \in S^{2}: \theta \neq \theta_{0}\right\}$ for $k \rightarrow \infty$. But unfortunately the behavior of $\sigma_{\gamma}$ for $\gamma>0$ and large values of $k$ is not investigated. Behavior of the scattering amplitude near the forward direction $\theta_{0}$ is unknown and therefore we don't have tools to calculate limits of measures like (8) , even if density $\varphi$ turns to zero at $\theta_{0}=0$, like it happens with Transport Cross section (5). But it becomes possible if we will prove that $\sigma_{\gamma}$ is bounded from infinity uniformly for all (large enough) values of $k$.

Theorem 1. 1. Exists a number $C=C(\gamma)>0$ such that for all $k \geq 0$

$$
\sigma_{\gamma} \leq C
$$

2. Let the visible part of $\Omega$ be written as a graph of the smooth function $g(x): \mathcal{I} \rightarrow \mathbb{R}^{3}$, where $\mathcal{I} \in \mathbb{R}^{2}$ is a part of plane perpendicular to $\theta_{0}$. Then

$$
\lim _{k \rightarrow \infty} R_{\gamma}=\int_{\mathcal{I}} \frac{2 d x}{1+|\nabla g|^{2}}\left(\frac{\gamma \sqrt{1+|\nabla g|^{2}}-1}{\gamma \sqrt{1+|\nabla g|^{2}}+1}\right)^{2} .
$$

Note that this integral has the form of a standard functional of the optimization theory. Cases $\gamma=0$ and $\gamma=\infty$ correspond to the classical resistance functional which was investigated starting from Newton (1686) (for example [9]-[12]) and in many recent articles. So, we suppose, that extremal problems of the constructed functional (12) is an object for investigation.

\subsection{Proofs}

The proof of Theorem 1 is based on the results of [1]. Let's prove the first part of the theorem 1: 
Proof. Since $f(\theta, k)$ is a continuous function on $k \geq 0$ (see [5]) we should prove that (11) holds for large values of $k$.

In [1, Prop. 2.1, p. 273] is proved that scattering amplitude has estimation

$$
\left|f_{\gamma}(\theta)-\tilde{f}_{\gamma}(\theta)\right| \leq \frac{C_{m}(\gamma)}{k^{m}}, \quad \theta \in S^{2}
$$

where $\left\{C_{m}(\gamma)\right\}$ are positive constants and $m$ can be chosen arbitrary large. Function $\widetilde{f}(\theta)$ is determined from the expression

$$
\widetilde{u}(r)=\left(e^{i k|r|} /|r|\right)(\tilde{f}(\theta)+o(1)), \quad r \rightarrow \infty .
$$

In own turn field $\widetilde{u}(r)$ is determined through

$$
\widetilde{u}(r)=\int_{\partial \Omega}\left(\widetilde{u}_{0}(t) \frac{\partial}{\partial n} \frac{e^{i k|r-t|}}{|r-t|}-\frac{\partial}{\partial n} \widetilde{u}_{0}(t) \frac{e^{i k|r-t|}}{|r-t|}\right) d S(t),
$$

where function $\widetilde{u}_{0}$ is at least twice differentiable in $\overline{\Omega^{\prime}}$ (see [1, formula 2.3'] for detailes) and therefore functions $\widetilde{u}_{0}(t)$ and $\frac{\partial}{\partial n} \widetilde{u}_{0}(t)$ are bounded on $\partial \Omega$.

¿From (14) we obtain that exists such $\widetilde{C}=\widetilde{C}(\gamma)$ such that for all $\theta \in S^{2}$ and values of $k$ large enough

$$
\left|\widetilde{f}_{\gamma}(\theta)\right|<\widetilde{C} k
$$

and due to (13) follows that the same bound (maybe with another constant $\widetilde{C}_{2}$ ) is valid for $f(\theta)$. Therefore, using optical theorem (see [3] for proof in case of impedance boundary condition )

$$
\sigma_{\gamma}=\frac{4 \pi}{k} \operatorname{Im}\left[f_{\gamma}\left(\theta_{0}\right)\right]
$$

we obtain that

$$
\sigma_{\gamma} \leq 4 \pi \widetilde{C}_{2}
$$

This ends the proof of the first part of the theorem 1 .

¿From (10) we have for $k \rightarrow \infty$

$$
\left|f_{\gamma}(\theta)\right|^{2}=\frac{1}{4} \mathcal{K}\left(y^{+}(\theta)\right)^{-1}\left(\frac{\gamma-<\boldsymbol{n}(\theta), \theta>}{\gamma+<\boldsymbol{n}(\theta), \theta>}\right)^{2}+O(1 / k), \quad \theta \neq \theta_{0},
$$

where estimation $O(1 / k)$ in (16) is uniform on compact subsets of $\{\theta \in$ $\left.S^{2} \mid \theta \neq \theta_{0}\right\}$. 
Using (15) and the fact that density of the integral (5) is continuous and it turns to zero in the point $\theta=\theta_{0}$, we obtain for $k \rightarrow \infty$

$$
\begin{aligned}
& R_{\gamma}=\int_{S^{2}}\left(1-<\theta, \theta_{0}>\right)\left|f_{\gamma}(\theta)\right|^{2} d \sigma(\theta)= \\
& \int_{S^{2}}\left(1-<\theta, \theta_{0}>\right)\left(4 \mathcal{K}\left(y^{+}(\theta)\right)\right)^{-1}\left(\frac{\gamma-<\boldsymbol{n}(\theta), \theta>}{\gamma+<\boldsymbol{n}(\theta), \theta>}\right)^{2} d \sigma(\theta)+o(1),
\end{aligned}
$$

Let us construct a change of variables $\theta(x): \mathcal{I} \rightarrow S^{2}$. Let $\boldsymbol{n}(x)$ be an outward normal in the point $y^{+}(x)=(x, g(x)) \in \partial \Omega$, then we put $\theta(x)=$ $\theta_{0}-2<\boldsymbol{n}(x), \theta_{0}>\boldsymbol{n}$. It's easy to see that $\theta(x) \in S^{2}$ and this map is one-to-one, since the obstacle $\Omega$ is strictly convex and therefore the Gauss map $\boldsymbol{n}(x)$ is also one-to-one.

Let us introduce standard spherical coordinates $(\cos (\widetilde{\theta}), \widetilde{\varphi}) \in[-1,1] \times$ $[0,2 \pi)$ and we will calculate Jacobian $D(\cos (\widetilde{\theta}), \widetilde{\varphi}) / D\left(x_{1}, x_{2}\right)$, where $\left(x_{1}, x_{2}\right)=$ $x$ are orthonormal coordinates on $\mathcal{I}$.

Note that

$$
\begin{aligned}
& \theta(x)=\left(\frac{2 g_{x_{1}}^{\prime}}{1+|\nabla g|^{2}}, \frac{2 g_{x_{2}}^{\prime}}{1+|\nabla g|^{2}}, \frac{|\nabla g|^{2}-1}{|\nabla g|^{2}+1}\right) \\
& \cos (\widetilde{\theta})=1-\frac{2}{|\nabla g|^{2}+1}, \\
& \widetilde{\varphi}=\arctan \left(g_{x_{2}}^{\prime} / g_{x_{1}}^{\prime}\right) \\
& \frac{D(\cos (\widetilde{\theta}), \widetilde{\varphi})}{D\left(x_{1}, x_{2}\right)}=\left|\begin{array}{ll}
\cos (\widetilde{\theta})_{x_{1}}^{\prime} & \widetilde{\varphi}_{x_{1}}^{\prime} \\
\cos (\widetilde{\theta})_{x_{2}}^{\prime} & \widetilde{\varphi}_{x_{2}}^{\prime}
\end{array}\right|= \\
& \frac{4}{\left(1+|\nabla g|^{2}\right)\left(|\nabla g|^{2}\right)}\left|\begin{array}{cc}
g_{x_{1}}^{\prime} g_{x_{1} x_{1}}^{\prime \prime}+g_{x_{2}}^{\prime} g_{x_{1} x_{2}}^{\prime \prime} & g_{x_{1}}^{\prime} g_{x_{1} x_{2}}^{\prime \prime}-g_{x_{2}}^{\prime} g_{x_{1} x_{1}}^{\prime \prime} \\
g_{x_{1}}^{\prime} g_{x_{1} x_{2}}^{\prime \prime}+g_{x_{2}}^{\prime} g_{x_{2} x_{2}}^{\prime \prime} & g_{x_{1}}^{\prime} g_{x_{2} x_{2}}^{\prime \prime}-g_{x_{2}}^{\prime} g_{x_{1} x_{2}}^{\prime \prime}
\end{array}\right|= \\
& \frac{4\left(\left(g_{x_{1} x_{2}}^{\prime \prime}\right)^{2}-g_{x_{1} x_{1}}^{\prime \prime} g_{x_{2} x_{2}}^{\prime \prime}\right)}{1+|\nabla g|^{2}}=-4 \mathcal{K}\left(x_{1}, x_{2}\right) \text {. }
\end{aligned}
$$

Note now that for $x \in \mathcal{I}$

$$
<\theta_{0}-\theta(x), \theta_{0}>=<2<\boldsymbol{n}(x), \theta_{0}>\boldsymbol{n}, \theta_{0}>=2<\boldsymbol{n}(x), \theta_{0}>^{2}=\frac{2}{1+|\nabla u(x)|^{2}},
$$

Applying (19) and (18) for (17) we obtain (12). Theorem 1 is proved. 


\section{References}

[1] A. Majda, High frequency Asymptotics for the Scattering matrix and the inverse problem of Acoustical scattering, Comm. pure and applied math. vol. XXIX, 261-291, (1976)

[2] A. Majda, M.E.Taylor, The asymptotic behavior of the diffractive peak in classical scattering, Comm. pure and applied math. vol. XXX, 639669, (1977)

[3] Kriegsmann GA, Alternate proof of the optical theorem for impenetrable targets, J Acoust Soc Am., 2006 Jan;119(1):31-2.

[4] Tychonov, A. N., and Samarsky, A. A., Equations of Mathematical Physics Pergamon, Oxford, 1963.

[5] Ramm A. G., Scattering by Obstacles (Dordrecht: Reidel), 1986

[6] A.I.Aleksenko, W. de Roeck, E.L.Lakshtanov, Resistance of the Sphere to a Flow of Quantum Particles, J.Phys. A.Math.Gen, (39), pp. 4251$4255,2005$.

[7] W. De Roeck, E.L.Lakshtanov, Total cross section exceeds transport cross section for quantum scattering from hard bodies at low and high wave numbers, J.Math.Phys, 48, 2007.

[8] Banchoff, T., Gaffney T., McCrory C., Cusps of the Gauss Map, (1982) Research Notes in Mathematics 55, Pitman, London.

[9] I. Newton, Philosophiae naturalis principia mathematica 1686.

[10] G. Buttazzo, B. Kawohl, On Newton's problem of minimal resistance, Math. Intell. 15, No.4, 7-12 (1993).

[11] A. Yu. Plakhov. Newton's problem of a body of minimal aerodynamic resistance. Dokl. Akad. Nauk 390, Nº3, 314-317 (2003).

[12] M. Comte, T. Lachand-Robert, Newton's problem of the body of minimal resistance under a single-impact assumption. Calc. Var. Partial Differ. Equ. 12, 173-211 (2001). 